\title{
Effective catalytic deoxygenation of palm fatty acid distillate for green diesel production under hydrogen-free atmosphere over bimetallic catalyst CoMo supported on activated carbon
}

\begin{abstract}
Palm fatty acid distillate (PFAD) is considered as an inedible and renewable feedstock for the production of green diesel. In the current study, green diesel was successfully synthesised via catalytic deoxygenation of PFAD in an environment free of $\mathrm{H} 2$ using a mesoporous activated carbon (AC) supported CoMo catalyst with various molybdenum (Mo) concentrations (5-20 $\mathrm{wt} \%$ ). Based on the study results, bimetallic catalyst Co10Mo10/AC formulation exhibited excellent catalytic performance with $92 \%$ hydrocarbon components (C8-C20) yield and 89\% selectivity for $\mathrm{n}-(\mathrm{C} 15+\mathrm{C} 17)$ with a total acid number of $24 \mathrm{mg} \mathrm{KOH} \mathrm{mg-1.} \mathrm{Based} \mathrm{on} \mathrm{a}$ comparison study with various supports (AC, $\gamma$-A12O3, TiO2), the AC-supported CoMo catalyst showed higher deoxygenation activity than both Co10Mo10/ $\gamma$-Al2O3 and Co10Mo10/TiO2 owing to the super acid-base sites as a result of synergism between the CoMo and AC support. The Co10Mo10/AC catalyst demonstrated excellent stability during the study as it maintained the hydrocarbon components yield and selectivity of $n-(\mathrm{C} 15+\mathrm{C} 17)>80 \%$ until the sixth run.
\end{abstract}

Keyword: Cobalt; Deoxygenation; Decarboxylation; PFAD; Molybdenum 